\title{
Correction to: Regulation of JAK/STAT signal pathway by miR-21 in the pathogenesis of juvenile idiopathic arthritis
}

\author{
Hong-Wei $\mathrm{Li}^{1,2} \cdot$ Hua-Song Zeng ${ }^{1,2}$ \\ Published online: 28 May 2021 \\ ๑) Children's Hospital, Zhejiang University School of Medicine 2021

\section{Correction to: World Journal of Pediatrics (2020) 16:502-513 https://doi.org/10.1007/s12519-019-00268-w}

The originally published version of this article contained some errors in (1) the graphs of Figs. $2 c, 2 f-j, 4$, and $6 b$; (2) the legends of Figs. 1-4, and 6. The corrected figures and legends are given in this Correction.

Fig. 2 Transfection of LV3-miR-21 suppressed p-STAT3/ STAT3 protein expression. a, b The titer of miR-21 in RASFs after LV3-miR-21 transfection; c OD value of RASFs with the simulation of IL-6 ( $* P<0.05$, IL-6 $100 \mathrm{ng} / \mathrm{mL}$ vs. control group, IL-6 150 ng/mL vs. control group); d-j The expression of STAT3/p-STAT3/SOCS3 in RASFs after transfection of LV3-miR-21 with the simulation of IL-6 (d $P<0.05$,
IL-6 + LV3-miR-21 vs. IL-6 + LV3-NC group; e $* P<0.05$, LV3-miR-21 vs. LV3-NC group, $\mathbf{f} * P<0.05$, IL-6+LV3-NC vs. IL-6 + control group, ${ }^{\dagger} P<0.05$, IL-6+LV3-miR-21 vs. IL-6 + LV3-NC group; $\mathbf{g} * P<0.05$, IL-6+ control vs. control group; $\mathbf{h} * P<0.05$, IL-6+LV3-miR-21 vs. IL-6 + control group, IL-6 + LV3-NC vs. IL-6 + control group; $\mathbf{i} * P<0.05$, LV3-miR-21 vs. control group, ${ }^{\dagger} P<0.05$, IL-6+LV3-miR-21 vs. LV3-miR-21 group; j $* P<0.05$, IL-6 + LV3-miR-21 vs. LV3-miR-21 group). STAT3 signal transducers and activators of transcription 3, SOCS3 suppressor of cytokine signaling 3, $I L-6$ interleukin-6, RASF rheumatoid arthritis fibroblast-like synovial cell, $N C$ negative control.

The original article can be found online at https://doi.org/10.1007/ s12519-019-00268-w.

Hua-Song Zeng

huasongxuqing@163.com

1 Department of Pediatric Allergy Immunology and Rheumatology, Guangzhou Women and Children Medical Center, Guangzhou 510623, China

2 Department of Pediatric Allergy, Immunology and Rheumatology, Guangzhou Women and Children's Medical Center, Guangzhou Medical University, 9 Jinsui Road, Guangzhou 510175, China 

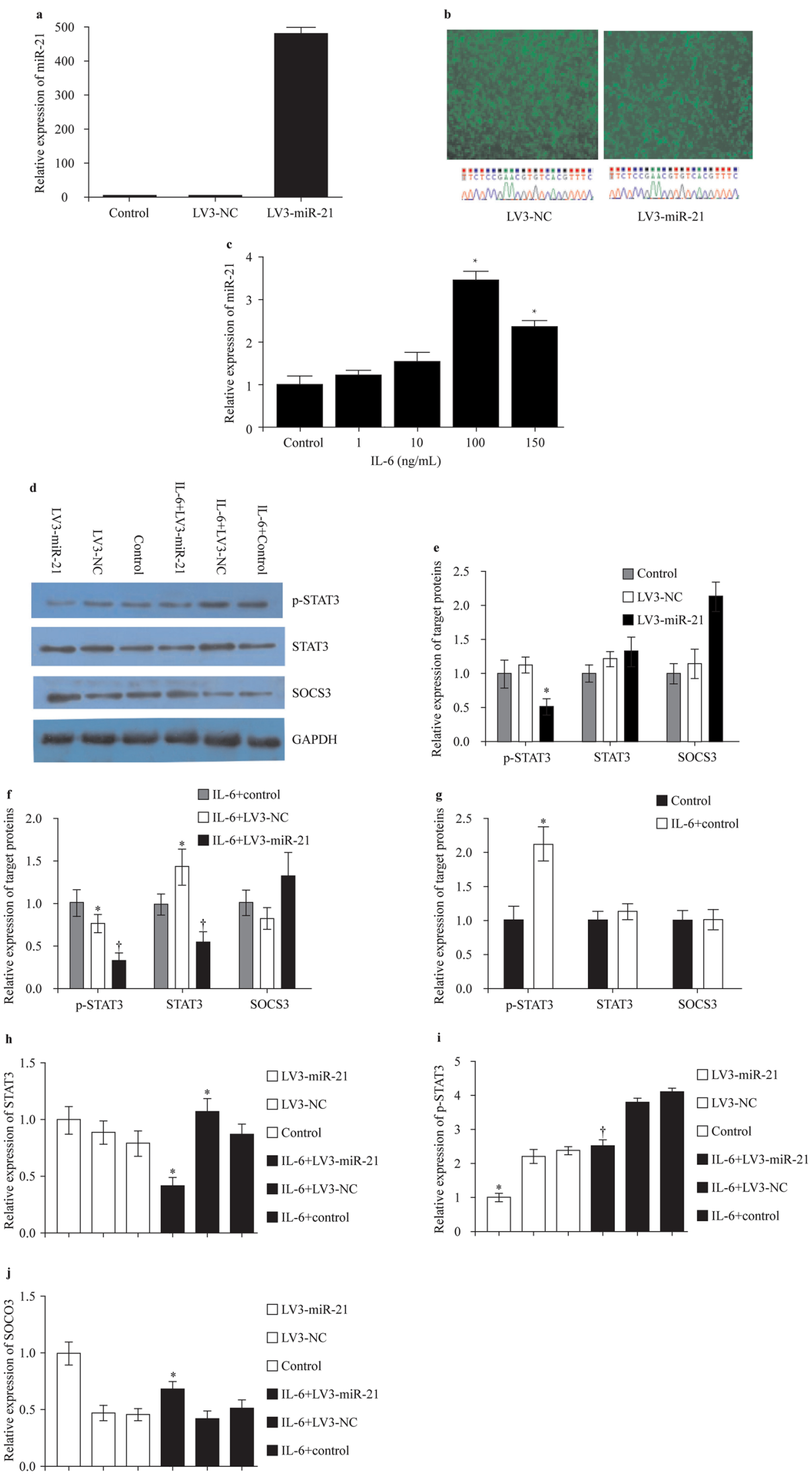
a
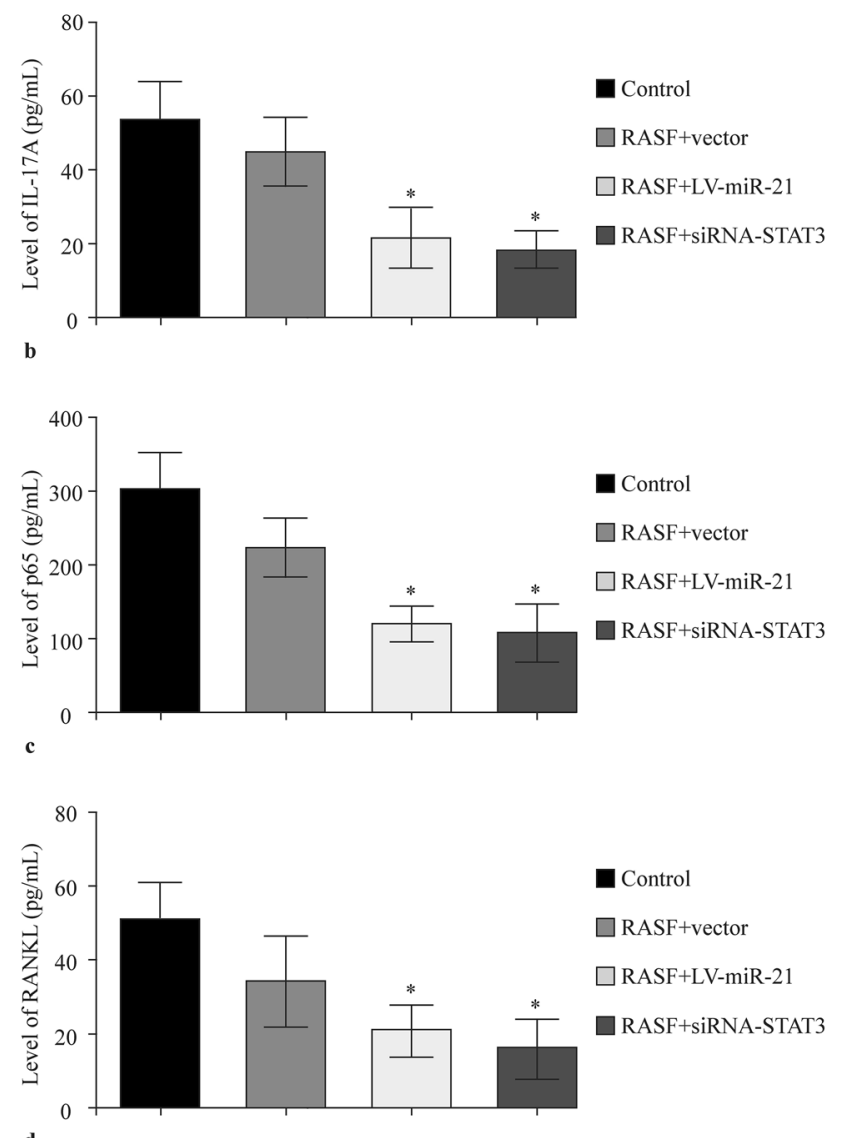

d

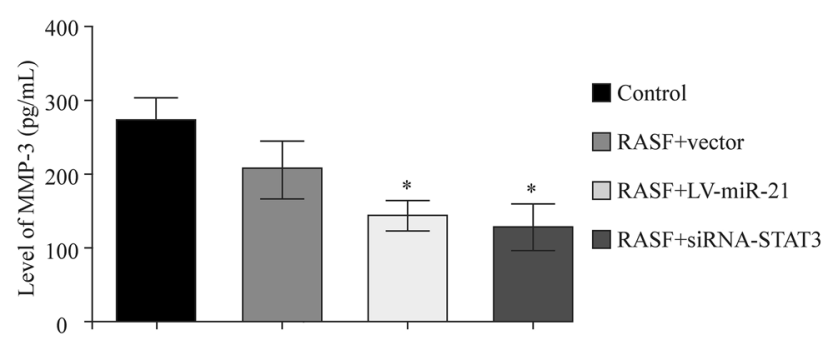

e

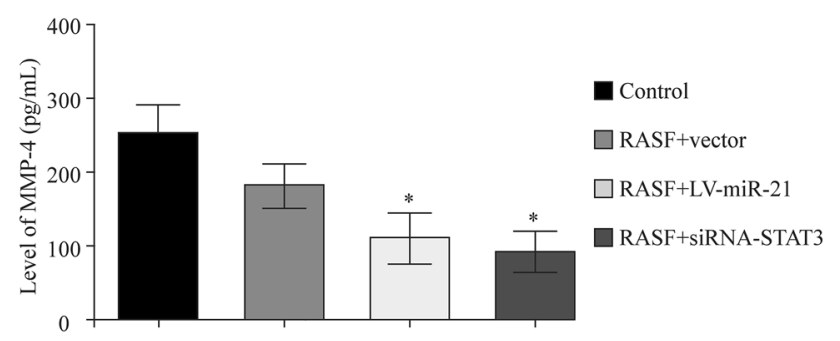

Fig. 4 Enhancing miR-21 or silencing STAT3 could suppress the expression of IL-17A (a), p65 (b), RANKL (c), MMP-3 (d), and MMP-4 (e). STAT3 signal transducers and activators of transcription 3, IL interleukin, $R A S F$ rheumatoid arthritis fibroblast-like synovial cell, $M M P$ matrix metalloproteinase, $R A N K L$ receptor activator of nuclear factor- $\mathrm{kB}$ ligand. $* P<0.05$, RASF $+\mathrm{LV}$-miR-21 vs. RASF + vector group, RASF + siRNA-STAT3 vs. RASF + vector group

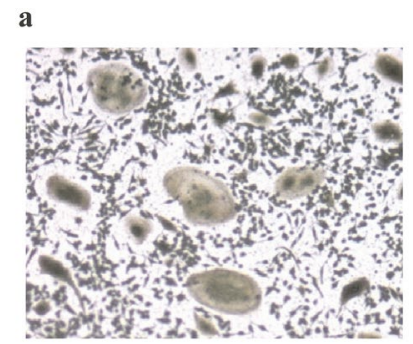

Control+M-CSF

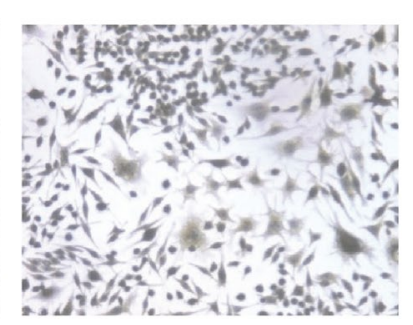

MiR-21+M-CSF

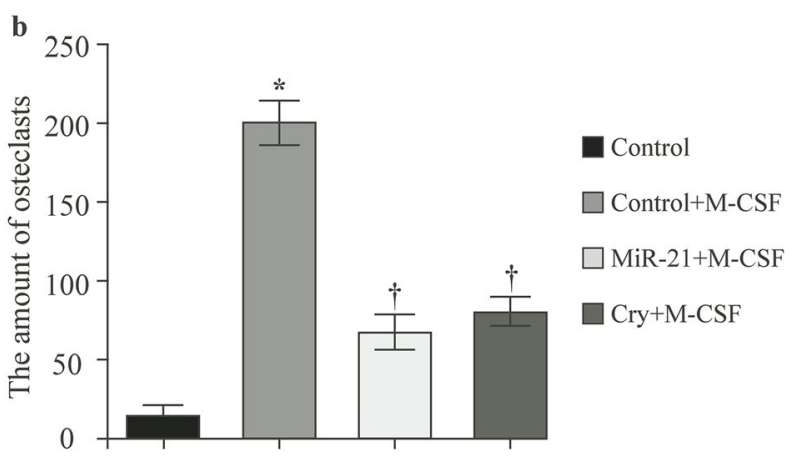

Fig. 6 M-CSF was used for inducing the RASFs to osteoclasts. a The TRAP staining results showed that the amounts of osteoclasts in miR-21 mimics group were significantly lower than the control group $(P<0.05$, miR- $21+\mathrm{M}-\mathrm{CSF}$ vs. control + M-CSF group); b Both miR-21 mimics and cryptotanshinone (cry) could decrease the amount of osteoclasts with no difference between these two groups ( ${ }^{*} P<0.05$, control $+\mathrm{M}$-CSF vs. control group, ${ }^{\dagger} P<0.05$, miR-21 + M-CSF vs. control + M-CSF group, cry + M-CSF vs. control $+\mathrm{M}$-CSF group). RASF rheumatoid arthritis fibroblast-like synovial cell, $M$-CSF macrophage colonystimulating factor

The corrected legends of Figs. 1 and 3 are as follows:

Fig. 1 Expression of miR-21 (a), STAT3 (b) and SOCS3 (c) mRNA of PBMCs and their correlation $(\mathbf{d}, \mathbf{e})$ in JIA. SJIA systemic juvenile idiopathic arthritis, $p J I A$ polyarticular juvenile idiopathic arthritis, STAT3 signal transducers and activators of transcription 3, SOCS3 suppressor of cytokine signaling 3, PBMCs peripheral blood mononuclear cells. $* P<0.05$, sJIA vs. control group, pJIA vs. control group

Fig. 3 Enhancing miR-21 or silencing STAT3 could suppress the IL-6 induced regulation of MMP-3 (a), MMP-4 (b), RANKL (c), and NF-kb (d). STAT3 signal transducers and activators of transcription $3, I L-6$ 
interleukin-6, RASF rheumatoid arthritis fibroblast-like synovial cell, MMP matrix metalloproteinase, RANKL receptor activator of nuclear factor- $\kappa \mathrm{B}$ ligand, $N F$ $\kappa b$ nuclear factor- $\kappa \mathrm{B} . * P<0.05, \mathrm{RASF}+\mathrm{LV}-\mathrm{miR}-21$ vs. RASF + vector group, RASF + siRNA-STAT3 vs. RASF + vector group
In the paragraph under the section "Results-Transfection of LV3-miR-21 suppressed p-STAT3/STAT3 protein expression": The sentence "Then RASFs were stimulated with IL-6 $(0,10,100,150 \mu \mathrm{g} / \mathrm{mL})$ for $4 \mathrm{~h}$ (Fig. 2c)" should read as "Then RASFs were stimulated with IL-6 $(0,10,100$, $150 \mathrm{ng} / \mathrm{mL}$ ) for $4 \mathrm{~h}$ (Fig. 2c)". 